\title{
Amenazas y vulnerabilidades: las dos caras de los desastres en Celestún, Yucatán*
}

\author{
Denise Soares, Daniel Murillo, \\ Roberto Romero y Gemma Millán
}

\begin{abstract}
Las condiciones sociales y ambientales de los riesgos, las respuestas frente a los desastres y la naturaleza de las relaciones entre desastres y desarrollo han sido objeto de creciente atención de investigaciones de las ciencias sociales porque los riesgos de desastres se han convertido en una amenaza en virtud del cambio climático. En este trabajo se reflexiona sobre la triada vulnerabilidad-desastres-desarrollo y se presenta un estudio de caso en el municipio de Celestún, Yucatán, sobre percepciones sociales acerca de la vulnerabilidad de la población frente a las amenazas de eventos meteorológicos extremos. Se pretende conocer las opiniones de la población respecto de su acceso a la capacitación sobre cómo enfrentar los huracanes, su capacidad para protegerse de dichas amenazas y su evaluación de la gestión de las autoridades locales en la materia.
\end{abstract}

PALABRAS CLAVE: vulnerabilidad, desastres, amenazas, riesgos, Celestún

\section{Threats and Vulnerabilities: Two Faces of Disasters in Celestún, Yucatán}

Social and environmental constraints of the risks, responses to disasters, as well as the nature of the relationship between disaster and development have been the subject of increasing attention from researchers in the social sciences. That's because the risks of disasters have become a growing threat by climate change. In this work we reflect over the triad vulnerability-disaster-development and present a case study in the municipality of Celestún, Yucatán, on social perceptions about the vulnerability against extreme weather events. We expected to know the views of the population regarding hurricanes, their access to the training and to evaluation of local authorities management.

KEYWORDS: vulnerability, disasters, threat, risks, Celestun

Denise SoAres: Instituto Mexicano de Tecnología del Agua, Jiutepec, Morelos, México denisefsoares@yahoo.com.mx

Daniel Murillo: Centro de Investigaciones y Estudios Superiores en Antropología Social-Distrito Federal, México danielmurillo2@yahoo.com.mx
Roberto Romero: Instituto Mexicano de Tecnología del Agua, Jiutepec, Morelos, México rromero@tlaloc.imta.mx

Gemma Millán: Instituto Mexicano de Tecnología del Agua, Jiutepec, Morelos, México gemmacrismm@hotmail.com

Desacatos, núm. 44, enero-abril 2014, pp. 159-177

Recepción: 20 de junio de 2012 / Aceptación: 16 de mayo de 2013

\footnotetext{
* Investigación realizada con fondos de la Secretaría de Educación Pública-Consejo Nacional de Ciencia y Tecnología, Ciencias Básicas.
} 


\section{INTRODUCCIÓN}

S i hasta mediados del siglo pasado el debate sobre la relación entre la vulnerabilidad social y los riesgos de desastres no ocupaba un lugar destacado en la agenda del desarrollo, en la actualidad se ha convertido en tema prioritario y punto de referencia y reflexión casi obligatorio. Esto se debe, entre otros factores, a la concepción de que el aumento en los niveles de vulnerabilidad social a nivel local, aunado al cambio climático global, genera condiciones propicias para un drástico incremento del número de desastres a mediano y largo plazos.

Por su ubicación geográfica, México es muy susceptible al embate de huracanes, los cuales azotan las zonas costeras e interactúan con condiciones de vulnerabilidad de la infraestructura urbana y vial, y ocasionan desastres de distinto grado. Además de esta fragilidad física se registra una gran vulnerabilidad social, resultado del crecimiento poblacional de los últimos años, de la alta marginación y de las deficiencias en la planeación de los servicios públicos. Asimismo, la poca efectividad de las políticas ambientales y sociales a nivel local tiene consecuencias importantes sobre la vulnerabilidad social y debilita la capacidad de respuesta de las poblaciones de más bajos recursos ante la emergencia de catástrofes. De esa forma, la manifestación del desastre varía de acuerdo con las características del espacio geográfico y las condiciones socioeconómicas de la población.

Según plantea Macías (1992), la noción de vulnerabilidad no es estática sino dinámica, en tanto lleva implícito un contenido temporal y espacial que remite a procesos causantes de la condición de susceptibilidad, toda vez que opera el cambio de una determinada condición - de vulnerabilidad- a otra —después de recibir el daño-. Por tanto, la vulnerabilidad no es una condición autocontenida, sino definida por las amenazas que determinan su grado de exposición. Es imprescindible aclarar que una amenaza no afecta de igual manera a los grupos sociales, es decir, aquellos con elevado poder económico residentes en regiones peligrosas no son igual de vulnerables que los pobres que habitan las zonas de peligro. Mientras los primeros tienen acceso a tecnologías antirriesgos y disponen de una elevada capacidad de recuperación, los segundos no cuentan con tecnología ni tienen capacidad de respuesta.

En este artículo pretendemos problematizar diversos ámbitos de la vulnerabilidad con el propósito de dilucidar las percepciones sociales sobre algunos aspectos de ésta en el plano local. El trabajo comprende cinco secciones: primera, un breve análisis de la relación entre vulnerabilidad, desastres y desarrollo; segunda, el marco metodológico que sustenta la investigación; tercera, una aproximación al municipio de Celestún, costa de Yucatán, desde el referente ambiental y socioeconómico; cuarta, percepciones sociales sobre vulnerabilidad frente a huracanes en el municipio de Celestún, y finalmente las conclusiones.

\section{CONSIDERACIONES EN TORNO A VULNERABILIDAD, DESASTRES Y DESARROLLO}

La vulnerabilidad social juega un rol determinante en la construcción de los desastres. Una amenaza natural, como un huracán, no tiene por qué convertirse en desastre. Gran parte del riesgo puede evitarse con la disminución de la vulnerabilidad. De esta manera, los desastres como consecuencia del paso de huracanes no son sólo el resultado de fuerzas naturales, sino de cuestiones de índole social y ambiental, como la falta de ordenamiento territorial y planeación urbana, la carencia de infraestructura adecuada y las prácticas ambientales inapropiadas - como la deforestación-, entre otros. La conformación de los desastres está íntimamente relacionada con el estilo de desarrollo y una reflexión seria sobre el tema implica inevitablemente establecer la relación entre los desastres, los problemas ambientales y la sustentabilidad o, en su caso, la insustentabilidad delmodelode desarrollo.Lainsustentabilidad ambiental, el subdesarrollo y la pobreza son causas 
inevitables de los desastres, de suerte que éstos son una consecuencia lógica del subdesarrollo (Lavell, 1996, 2005).

Antes de continuar con los argumentos acerca de la conformación de los desastres es pertinente hacernos tres preguntas: ¿qué son las amenazas?, ¿qué es un desastre? y ¿qué es la vulnerabilidad? Concebimos las amenazas como fenómenos extremos, de origen natural o no, que actúan en un determinado espacio geográfico en un tiempo definido, con el potencial de causar daño a una población. Las amenazas "naturales" se refieren a fenómenos atmosféricos, hidrológicos, geológicos o incendios, los cuales por su ubicación, intensidad y frecuencia tienen elevada probabilidad de afectar adversamente a grupos sociales determinados, a sus actividades y a la infraestructura existente. En este documento nos ocuparemos de las amenazas "naturales" constituidas por los huracanes. Si bien le damos el apellido "natural" a algunas de éstas, el término "amenaza" contiene elementos sociales, dado que en regiones deshabitadas los fenómenos naturales no constituyen amenazas y tampoco conducen a desastres. Así, es justamente el componente humano el que convierte un fenómeno natural, como un huracán, en una amenaza natural (OEA, 1991). En relación con la segunda pregunta, según Wilches-Chaux, desastre es:

un evento identificable en el tiempo y en el espacio, en el cual una comunidad ve afectado su funcionamiento normal con pérdidas de vidas y daños de magnitud en sus propiedades y servicios, que impiden el cumplimiento de las actividades esenciales y normales de la sociedad (Wilches-Chaux, 1993: 12).

El autor añade que un desastre es una combinación de riesgo y vulnerabilidad y que el riesgo puede definirse como "cualquier fenómeno de origen natural o humano que signifique un cambio en el medio ambiente que ocupa una comunidad determinada, que sea vulnerable a ese fenómeno" (Wilches-Chaux, 1993: 17). Nos parece pertinente establecer los límites de tres conceptos que suelen homologarse en el debate sobre los riesgos: amenaza, riesgo y desastre. Las amenazas son fenómenos que tienen el potencial de afectar negativamente a los seres humanos. Los riesgos se refieren a las condiciones socioeconómicas y ambientales de la región que ponen al grupo social en condición de peligro ante la posibilidad de la amenaza. Y finalmente el desastre se concreta a partir de la interacción entre la amenaza y las condiciones de riesgo socialmente construidas que han incrementado las condiciones de vulnerabilidad de un cierto grupo social (García, 2005). Para responder a la pregunta relativa a la vulnerabilidad, Barriga (2004) plantea que la vulnerabilidad social está determinada por múltiples factores, algunos de los cuales se relacionan con políticas, instituciones y carencia de activos, y se expresa en condiciones de fragilidad o falta de resiliencia que predisponen a una comunidad o a un grupo o grupos de individuos por sus condiciones políticas, sociales, económicas, de edad, género y etnia a sufrir daño diferencial por estar expuestos ante una amenaza que genera un desastre, como los huracanes.

Según Lavell (2000), hay dos paradigmas contrapuestos para analizar los desastres: el físico o natural y el social. La interpretación que el paradigma físi$\mathrm{co} /$ natural brinda sugiere que los desastres son producto de extremos de la naturaleza que impactan a una sociedad. De hecho, esta idea se ve reflejada en la nomenclatura que se utiliza popularmente al hablar de desastres "naturales" o en la conversión del evento natural en el desastre mismo. De esa manera se introduce la noción de una cierta inevitabilidad en la concreción de los desastres, al ser actos de la naturaleza. El autor menciona que después del huracán Mitch, ubicado en la categoría cinco en la escala Safir-Simpson, que azotó Centroamérica en 1998, no faltaron quienes concluyeran desde una posición fatalista o naturalista que frente a la magnitud y la intensidad del acontecimiento no había mucho por hacer para reducir su impacto.

Por otro lado está el paradigma social, defendido, entre otros estudiosos del tema, por Lavell (2000, 2005), Wilches-Chaux (1989, 2000), Uribe et al. 
(1999), Adger (2006) y Blaikie et al. (1998), quienes argumentan que a pesar de la génesis natural del desastre éste presenta un fuerte componente político y social, es decir, los desastres ocurren como consecuencia de decisiones políticas previas, relacionadas con incorrectas o insuficientes políticas sociales, económicas y ambientales. Los autores se refieren al desastre como un problema no resuelto del desarrollo, al plantear el riesgo como un producto de las modalidades de desarrollo padecidas en Occidente en general y en la región latinoamericana en particular, basadas en una lógica de dominio de la naturale$\mathrm{za}$, que ha generado una crisis social y ambiental con sus consecuencias en términos de empobrecimiento de grandes masas de población, desigualdad y exclusión social, contaminación de fuentes de agua, pérdida de la biodiversidad, etc. Los modelos de desarrollo se asocian con las expresiones de riesgo y vulnerabilidad, de modo que el círculo vicioso del desastre está profundamente inserto en el del subdesarrollo y la pobreza. En resumen, el problema bras de Luhmann (1992), la sociedad actual es la sociedad del riesgo.

Un desastre no es un evento natural de origen geológico o meteorológico, como un sismo o huracán, sino que se constituye por los efectos que éstos producen en la sociedad. Los eventos naturales son un prerrequisito para que sucedan los desastres, pero en sí no son suficientes para que éstos se materialicen. Debe haber grupos sociales vulnerables a los impactos de los eventos naturales para que los mismos se conviertan en desastres. En opinión de Wilches-Chaux (2000), según la forma particular de desarrollo productivo, territorial, institucional, cultural, político, ambiental, social y de infraestructura, una comunidad resulta capacitada o incapacitada para absorber o recuperarse autónomamente de los impactos de los eventos físicos. La vulnerabilidad es una variable endógena del proceso de desarrollo y por ello resulta paradójico pensar en reducirla sin plantearse un cambio en el modelo en sí (Lavell, 2005).

Es muy difícil creer que el modelo de desarrollo, que conduce continuamente a la producción de pobreza, pueda tener una política social que garantice que los pobres vivan en condiciones de seguridad ambiental, en terrenos libres de riesgo y con condiciones de vida seguras. De esa manera, los desastres integran los procesos de desarrollo y son el precio a pagar por perseverar en el impulso de un modelo de crecimiento que garantiza bienestar para pocos y pobreza y vulnerabilidad para muchos. El crecimiento económico, concentrado en determinados sectores privilegiados, genera las condiciones para que suceda el desastre y por ello un balance objetivo de la ecuación desastre-desarrollo debe sustraer de las ganancias económicas aquellas logradas con la producción de la vulnerabilidad y las pérdidas asociadas con esta misma vulnerabilidad una vez concretado el desastre. Desafortunadamente, las ganancias generadas con la producción de la vulnerabilidad son privadas, mientras que la vulnerabilidad y las pérdidas sufridas durante los desastres son socializadas (Lavell, 1993, 1996, 2005).

Para atacar las condiciones que generan la vulnerabilidad y crear de esa manera sociedades con mayor capacidad de resiliencia, por definición el desarrollo tendría que vincular la mejoría de la calidad de vida de las poblaciones, la equidad social, la participación ciudadana efectiva en la toma de decisiones, la conservación y recuperación de los sistemas ecológicos. Habría que promover el crecimiento no sólo cuantitativo sino cualitativo, con poco impacto en el ambiente por intermedio de la implementación de nuevas formas de producción y la optimización de la distribución social de sus beneficios (Leff, 1993). Sin embargo, en Latinoamérica seguimos concibiendo el desarrollo como sinónimo de crecimiento económico de unos cuantos, lo que profundiza las desigualdades sociales, políticas y económicas, y produce y alimenta la vulnerabilidad de manera constante. Es interesante examinar la interpretación dada por Uribe y colaboradores al desastre provocado por Mitch en Centroamérica, desde una perspectiva social y no natural, como la planteada anteriormente: 
este desastre no fue tan sólo resultado de fuerzas naturales, sino también del comportamiento humano; los daños se aumentaron debido a prácticas descontroladas como el manejo incorrecto de las cuencas hidrográficas, el abuso de los recursos naturales, incluyendo la deforestación, la agricultura inadecuada de laderas sin tecnificar y la expansión urbana descontrolada (Uribe et al., 1999: 5).

Es inevitable la referencia a la vulnerabilidad como una de las causas de los desastres. Mientras los procesos de transformación social y crecimiento poblacional no garanticen una adecuada relación con el ambiente natural que le da sustento, los desastres seguirán en aumento. De esa manera la reducción de la vulnerabilidad requiere cambios fundamentales en las estrategias de desarrollo vigentes en la región. Sin embargo, se sigue apostando en la lógica prevaleciente en los modelos de desarrollo y considerando la vulnerabilidad y el riesgo como meras externalidades. $\mathrm{Al}$ respecto, Lavell afirma:

Resulta más fácil negar la naturaleza estructural de la vulnerabilidad y el riesgo, explicándola como un factor colateral, desatendido, olvidado o soslayado de los modelos de crecimiento, que asumir las consecuencias que su causalidad estructural exigiría. Es más fácil confortarse apoyando el rumbo impuesto con los modelos, buscando mejorarlos, introduciendo mecanismos cosméticos y efímeros para reducir el riesgo, que asumir el reto de la vulnerabilidad como algo estructuralmente determinado. Si el riesgo no está estructuralmente determinado, entonces puede reducirse haciendo ajustes al margen (Lavell, 2005: 20).

Si bien la interpretación de las causas de los desastres puede generar polémica y obedecer a distintas posturas, los datos duros en torno al impacto de los eventos naturales sobre las poblaciones son incuestionables y arrojan que las poblaciones más pobres son las más afectadas. Según Quinceno (2005), durante los últimos 25 años han muerto más de tres millones de personas debido a desastres y $90 \%$ de dichos eventos ha ocurrido en países del "tercer mundo”. En el mismo orden de ideas, Saavedra (1996) agrega que la tasa de mortalidad por desastres en los países pobres rebasa diez veces la misma tasa en los países ricos.

Una vez realizado este breve recorrido por los trabajos de algunos autores emblemáticos que estudian la triada vulnerabilidad-desastres-desarrollo, retomamos elementos de esas proposiciones conceptuales para conformar nuestro propio acercamiento al tema. Para nosotros la vulnerabilidad supone la incapacidad de determinados grupos sociales para hacer frente, resistir y recuperarse de eventos extremos y por ello es un elemento clave en la ecuación del desastre. Se puede afirmar que mantiene una relación directamente proporcional a éste, es decir, a mayor vulnerabilidad de los grupos sociales, mayor será el desastre resultado de la amenaza. La vulnerabilidad es multidimensional, en tanto involucra componentes socioeconómicos, ambientales y físicos. De la misma manera que la vulnerabilidad está estrechamente relacionada a los desastres, éstos son consecuencias de la carencia de desarrollo, de suerte que el fortalecimiento de los procesos de planeación del desarrollo tendrá un impacto positivo tanto en la reducción de los desastres como en la disminución de las vulnerabilidades. Una vez aclarada nuestra posición frente a los conceptos mencionados, expondremos el marco metodológico que ha orientado la investigación y enseguida nos acercaremos al municipio de estudio, ubicado en la costa yucateca, desde los referentes ecosistémico, demográfico, económico y de acceso a los servicios.

\section{MARCO METODOLÓGICO}

Para el desarrollo de este estudio se estableció una metodología compuesta por tres fases: 1) diseño de la investigación y construcción del marco teórico; 2) obtención de información empírica, y 3) análisis de los datos obtenidos y elaboración de conclusiones. En la primera etapa consultamos fuentes de información secundaria y revisamos bibliografía sobre los temas de amenazas, vulnerabilidad, riesgos de desastres 


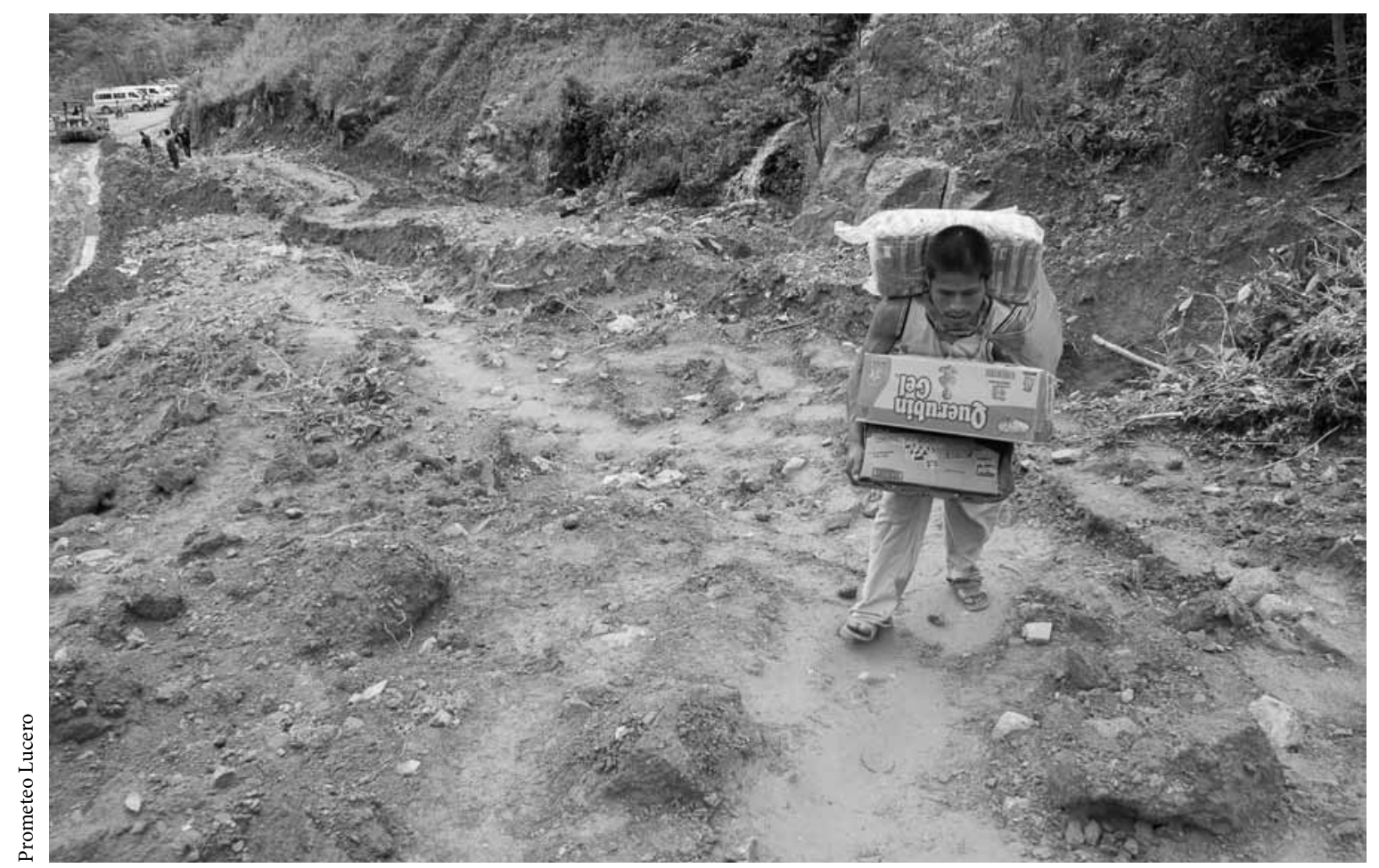

Las personas se transportan a pie por encima de los escombros de un derrumbe sobre la carretera Tlapa-Marquelia, a la altura del poblado de Tilapa, septiembre de 2010.

y su articulación con el desarrollo. Se estableció la segunda etapa, de trabajo de campo, para generar información empírica con la aplicación de encuestas y entrevistas como herramientas básicas para la obtención de información primaria, relativa a percepciones sociales sobre la vulnerabilidad frente a las amenazas de huracanes en el municipio de Celestún.

El universo de estudio para las encuestas fue de 182 personas residentes en Celestún -91 mujeres y 91 hombres-. Las personas encuestadas fueron mayores de 18 años y asumían el rol de jefes o jefas de familia. La muestra fue aleatoria y es estadísticamente representativa, toda vez que conforma $10 \%$ de las viviendas habitadas de la localidad - de un total de 1798-. El diseño de la encuesta contempló la definición de variables relacionadas con la identificación de las personas informantes y su percepción relativa a las amenazas y vulnerabilidades, de manera que cada variable por sí misma o en interacción con otras brindara la información que permitiera dar respuesta a los objetivos del estudio.

Las entrevistas semiestructuradas fueron aplicadas a 23 informantes clave, 20 residentes en Celestún y tres en Mérida. Las personas entrevistadas en Mérida son funcionarias de la Unidad Estatal de Protección Civil (PCE) y nos ha parecido relevante incluirlas en el estudio por su estrecha vinculación con el municipio en términos de brindar capacitación para enfrentar las amenazas de los huracanes. La recolección de los datos primarios se llevó a cabo de junio a noviembre de 2011. La entrevista como método cualitativo permite cruzar la información obtenida de manera cuantitativa y arrojar mayores elementos de análisis. En la tercera fase, de análisis de los datos, se construyó una base de datos para la encuesta, se realizó el tratamiento estadístico con el programa spss y se arribó a la integración de los resultados y las conclusiones. 


\section{UN ACERCAMIENTO AL MUNICIPIO DE CELESTÚN}

El pueblo de Celestún fue fundado en 1718, como una localidad dependiente de Sisal. Sin embargo, por ser una región pantanosa, el lugar permaneció casi despoblado. Al suprimirse el Partido de Sisal en 1872, pasó a formar parte de Maxcanú hasta 1918, cuando la población se erigió como cabecera del municipio de Celestún. A partir de 1970 se ha dado un fuerte impulso al incremento de la población de Celestún debido a la crisis henequenera, que ha derivado en políticas estatales que se orientaron a desarrollar la actividad pesquera como una estrategia para afrontar la crisis, lo que favoreció los desplazamientos de la población hacia la región costera, con acentuada migración durante la temporada de captura del pulpo (Conanp, 2002).

Celestún se ubica a 98 kilómetros al poniente de Mérida, capital del estado de Yucatán. Al norte limita con el Golfo de México, al oriente con Hunucmá, al poniente con el estado de Campeche y al sur con Kinchil (Fraga, 1993). Las principales actividades productivas de Celestún han estado históricamente vinculadas a la extracción de sal y a la pesca. Durante más de dos siglos la explotación de la sal fue el eje de la economía celestunense, al punto de que la actividad determinaba su crecimiento demográfico y reflejaba los periodos de auge o depresión de la industria salinera, que atraía o expulsaba a los trabajadores. La producción de sal local ha declinado desde 1940, en virtud de la instalación de una salinera moderna en Las Coloradas - municipio de Río Lagartos-, que derivó en el monopolio de la sal en la región. De manera paralela a la producción de sal, se practicaba la pesca, la cacería y la producción de copra (Uc, 2007).

Se han considerado cuatro rubros en el acercamiento al municipio de Celestún: variables ecosistémicas, demográficas, participación económica y niveles de acceso a los servicios. En lo que corresponde a las variables ecosistémicas, se menciona la relevancia de su estero, algunas especies de fauna $y$ flora con gran importancia ecológica y las oportunidades económicas dada la elevada diversidad biológica. En relación con las variables demográficas se especifican la población por sexo y el índice de masculinidad del municipio. Esto abre precedentes para indagatorias acerca de los niveles de migración y su impacto en la economía y la calidad de vida regional. En lo tocante a la participación económica se desglosan la población económicamente activa (PEA) ocupada por sexo y por sector. Finalmente, los niveles de acceso a los servicios son presentados como hombres y mujeres alfabetizados, acceso a los servicios de salud, número de viviendas con acceso a agua, drenaje, electricidad y escusados.

\section{Variables ecosistémicas}

La riqueza ecosistémica de Celestún se debe en gran parte a su ubicación privilegiada entre dos cuerpos de agua: por un lado el Golfo de México y por el otro un estero con una extensión de casi 22.5 kilómetros. La relevancia del estero reside en que es una zona de refugio, crianza, reproducción y alimentación de una gran variedad de peces, moluscos y crustáceos. Entre las principales especies económicas y ecológicas que habitan el estero encontramos el camarón (Farfantepenaeus spp.), la jaiba azul (Callinettis sapidus), el bagre (Arius melanopus) y la mojarra (Cichlasoma urophthalmus). En cuanto a comunidades vegetales del ecosistema celestunense existen dunas, manglares, petenes, selva baja inundable, pastizal, selva baja caducifolia y vegetación subacuática. Sobre la fauna, de las 554 especies reportadas, 15 son endémicas de México y dos de Yucatán. Hay 333 especies de aves, de las cuales 177 son residentes, 142 migratorias y 17 presentan poblaciones de ambos tipos, entre las que destaca el flamenco rosado (Phoenicopterus ruber ruber), una de las principales atracciones turísticas del municipio. Además, ahí se ubica parte de la desembocadura de la cuenca noroccidental de agua subterránea de la Península de Yucatán y su ecosistema integra el 
corredor costero de humedales mejor conservados de la península. En virtud de la relevancia ecológica de Celestún, en 2002 fue declarado Reserva de la Biosfera (Batllori, 2003; Conanp, 2002). ${ }^{1}$

\section{Variables demográficas}

Durante los últimos 40 años la presión demográfica sobre el territorio de Yucatán se ha más que duplicado: la densidad promedio de habitantes creció de $19.28 \mathrm{hab} / \mathrm{km}^{2}$ en 1970 hasta $49.48 \mathrm{hab} / \mathrm{km}^{2}$ en 2010. Y aunque actualmente el crecimiento poblacional en Yucatán es moderado desde el punto de vista estadístico, la tasa de crecimiento anual estimada entre 2000 y 2010 fue de 1.5 (INEGI, 2011). La región costera no es ajena a la presión demográfica: en los últimos 40 años ha experimentado un acelerado crecimiento poblacional, directamente relacionado con la caída de la actividad henequenera en el estado de Yucatán. De hecho, muchas familias de la zona henequenera se instalaron en la costa para dar inicio a su nueva actividad de subsistencia: la pesca. El crecimiento demográfico conlleva impactos variados para los ecosistemas estuarinos, con efectos nocivos para la población humana, al incrementar su vulnerabilidad frente a los eventos meteorológicos extremos, toda vez que actúan como una barrera que protege a las poblaciones de los vientos y subidas de marea.

El crecimiento poblacional de Celestún no se encuentra al margen de la tendencia experimentada en el resto de la región costera de Yucatán, y es inclusive potencialmente mayor por la conjugación de dos factores: por un lado ha recibido inmigrantes debido a la caída de la actividad henequenera en el estado y por el otro se ha transformado en un centro turístico de relevancia nacional, por sus atractivos naturales. Prueba de ello es su vertiginosa dinámica

\footnotetext{
${ }^{1}$ La Reserva de la Biosfera de Celestún cuenta con 81482.33 hectáreas y abarca los municipios de Celestún y Maxcanú, en Yucatán, y Calkiní, en Campeche (Conanp, 2002).
}

poblacional: de alrededor de 1000 habitantes en 1950 a casi 7000 en 2010. En 2010 se reportó en Celestún una población total de 6810 habitantes, entre éstos 3326 mujeres y 3484 hombres (INEGI, 2011).

En el municipio de Celestún la movilidad de personas que buscan oportunidades de trabajo es elevada, a raíz de su pujante actividad turística, cuyas principales atracciones son el avistamiento de aves, en especial los flamingos, y la pesca deportiva. El crecimiento poblacional no ha contado con la debida planeación, se han construido viviendas en zonas inadecuadas, como el mangle o la ciénaga, lo que representa un peligro no sólo en términos de destrucción de la biodiversidad, sino de incremento de la vulnerabilidad social. Es imprescindible planificar el crecimiento de Celestún para que no cambie el uso de suelo en detrimento de la biodiversidad local y la agudización de los riesgos de desastres para la población.

En lo referente al índice de marginación, medido como la intensidad de las privaciones de la población en dimensiones relativas a la educación, la vivienda y los ingresos monetarios, Celestún presenta una marginación media -25.046 en una escala del uno al 100-, ocupa el lugar 85 en el contexto estatal y el 1397 a nivel nacional (Conapo, 2011). Uno de los componentes de la vulnerabilidad es justamente la marginación, ya que integra las carencias de la población y su capacidad para enfrentarse a amenazas (Barrachena et al., 2000).

\section{Participación económica}

Cuantificar la PEA de la región de estudio nos ayuda a construir una visión de la economía de la zona. Para acercarnos con mayor certidumbre a la dinámica de dicha economía nos interesa enfocarnos en la población ocupada, dado que es la que genera ingresos en las comunidades. El porcentaje de población ocupada en 2010 en la localidad de Celestún fue de 39\%. Como era de esperarse, debido a la división sexual del trabajo, en la que los hombres desempeñan 
las tareas de proveedores, éstos predominan en este rango $-31 \%$ - El hecho de que pocas mujeres sean económicamente activas - $8 \%$ - indica una mayor vulnerabilidad para este grupo de población ante un desastre por su dependencia económica, pero también por la marginalidad que ha vivido para desarrollar capacidades y habilidades en un sentido distinto del doméstico y de cuidado (INEGI, 2011). En términos de población ocupada asociada al sector productivo, la pesca concentra poco menos de $50 \%$, mientras que la población ocupada en el sector terciario es de $40.17 \%$. Celestún es el principal centro turístico de la costa yucateca y el número de personas que se dedican a la prestación de servicios turísticos tiende a incrementar. Celestún es el segundo municipio de mayor importancia después de la salinera de Río Lagartos (INEGI, 2011), con $12.45 \%$ de su población ocupada en el sector secundario de la industria de extracción de sal.

Hasta hace poco tiempo la economía costera en Yucatán se basó en la subsistencia y capitalización por excedentes hacia los mercados local y regional. Esta lógica respondía al desarrollo del Estadonación; sin embargo, el desarrollo de ciertos polos y ciudades, así como una demanda internacional de productos marinos rebasaron esta dinámica, lo que generó que la posibilidad local de desarrollo se insertara en un contexto de globalización. Aunado a esto, la sustitución del henequén por productos sintéticos y el abandono de actividades agropecuarias agudizaron la situación económica regional basada en la actividad primaria de tipo campesino y de pescador de pequeña escala y de subsistencia. Así, la actividad productiva cambió hacia la de servicios, en particular la construcción, que ocupa un lugar relevante en la economía local. La pesca pasó a ser la actividad productiva primaria preponderante y la que mayores ingresos genera, por lo que se ha abandonado el campo. No obstante, esta transición no consolidó la actividad pesquera, sino que siguió el patrón oligopólico del Estado, en el que unos cuantos permisionarios privados acapararon el acopio, el procesamiento y la comercialización de los productos marinos (Canto, 2001). La vulnerabilidad que hoy se presenta en la población es causada por la precariedad del empleo y falta de capacitación de la población. Un posible desastre traería consecuencias de extrema pobreza en la costa de Yucatán y problemas socioeconómicos muy graves por la falta de apoyo y de prioridad en cuanto a la estructura productiva dominante en la costa. La población difícilmente podría asimilar desastres con sus condiciones económicas actuales.

\section{Nivel de acceso a los servicios en las viviendas}

El acceso a una vivienda digna se ha identificado como una necesidad humana básica y como un aspecto importante en la autonomía personal y comunitaria que facilita el desarrollo y la realización personal (Salles y López, 2004). El desarrollo urbano sin planeación, la proliferación de casas en el litoral, el establecimiento y la construcción de infraestructura portuaria y carretera han dañado severamente la contaminado e interrumpido los flujos de agua, lo que vulnera tanto a los ecosistemas como a los pobladores frente a los fenómenos meteorológicos, como los huracanes. $\mathrm{Al}$ acercarnos a las viviendas de la zona de estudio, encontramos que el acceso a los servicios es desigual. Mientras la electricidad es el servicio más accesible para las viviendas - $94.09 \%$-, se observan fuertes rezagos en el drenaje $-73.61 \%$ de cobertura-y aún hay $15 \%$ de viviendas sin escusado sanitario y $10 \%$ sin agua entubada. Es necesario aclarar que en la región costera de Yucatán el suelo de tipo calcáreo y la poca profundidad del manto freático limitan la obra de drenaje convencional, por lo cual se considera drenaje la conexión de la vivienda a una fosa séptica. La carencia de drenaje plantea la posible existencia de un fuerte problema de contaminación de los suelos y mantos acuíferos, situación que podría derivar en graves desequilibrios en la vida acuática y complicaciones de salud a 
los seres humanos. En tanto los municipios no realicen acciones encaminadas a dar una solución aceptable a un problema de dimensiones crecientes, dicho tema será de polémica (INEGI, 2011).

El rezago de acceso a los servicios que presenta Celestún en términos generales es atribuible al rápido crecimiento poblacional y a la falta de estrategias consistentes de planeación urbana. De mantenerse la tendencia actual de crecimiento urbano desordenado, el futuro cercano supone un gran reto de acceso a los servicios básicos, dado que dicho crecimiento es caótico, sin ningún plan de desarrollo urbano ambientalmente sustentable para satisfacer los requerimientos de la población. La salud es un problema constante en la región de estudio y su población se enfrenta con frecuencia a enfermedades de la pobreza, como diarreas y parasitosis, consecuencia de las condiciones de insalubridad. A pesar de las deficiencias sanitarias de la región, no existe un plan de salud capaz de contrarrestar las carencias de la población. De hecho, el acceso a la salud es un nudo en lo que a servicios se refiere. En ese sentido la media de la población con derecho a servicios de salud -70\% - es baja si la comparamos con otros servicios, como el agua o la electricidad (INEGI, 2011).

La educación condiciona la calidad de vida de un individuo. La alfabetización es una necesidad básica de aprendizaje y se relaciona con la ciudadanía, la identidad cultural, el desarrollo socioeconómico, los derechos humanos y la equidad (UNESCO, 2006). Los datos del Censo de Población y Vivienda 2010 muestran que $6.65 \%$ de la población de Celestún con 15 años y más es analfabeta, en tanto que el analfabetismo por sexo arroja que la tasa de analfabetismo es mayor entre los hombres que entre las mujeres -239 hombres y 214 mujeres (INEGI,

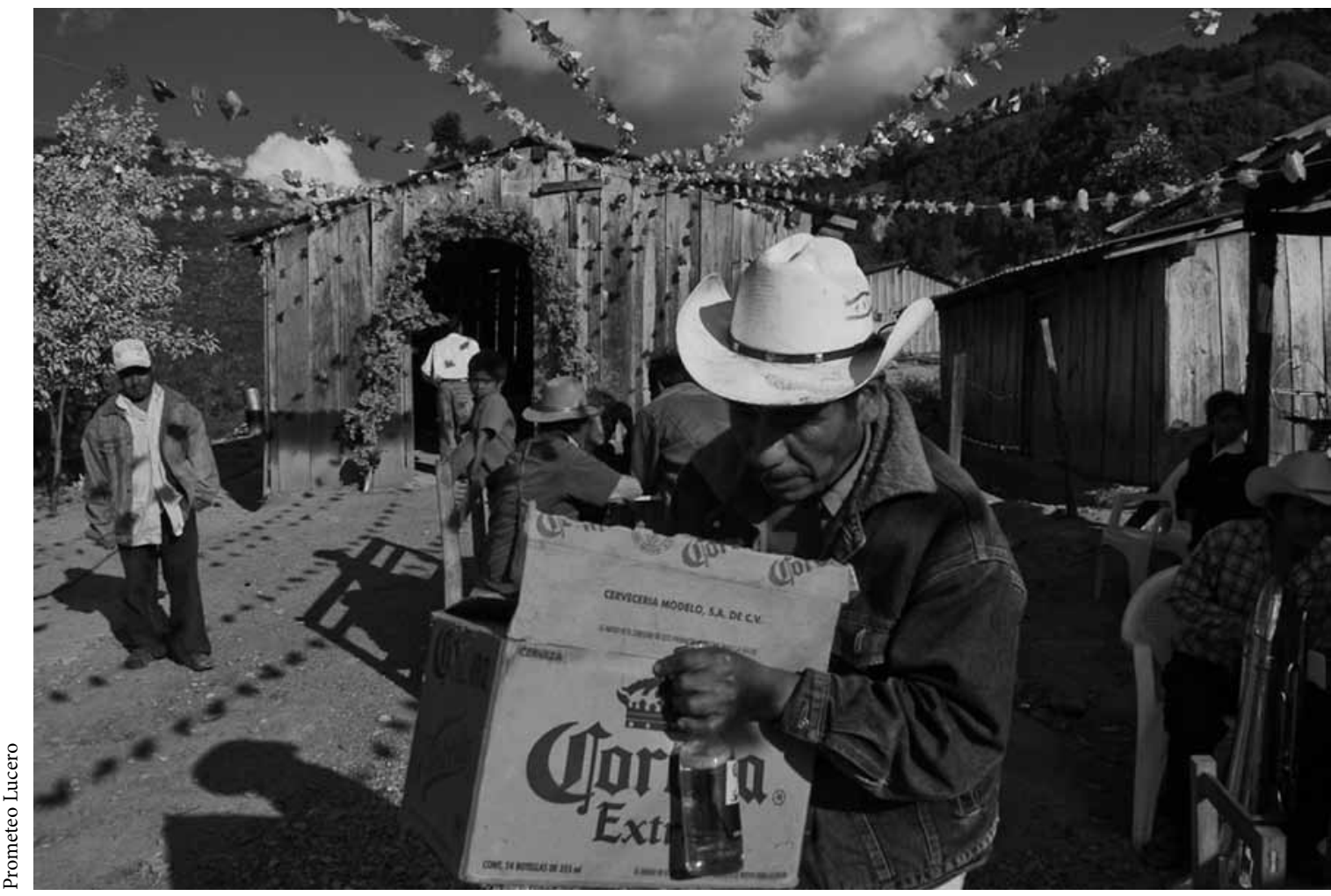

Conmemoración de la fundación de La Barca, comunidad erigida después de que un sismo destruyera las viviendas, abril de 2010. 
2011) - El analfabetismo influye en el hecho de que la población no esté preparada para resistir de la mejor manera un posible desastre, lo que implica problemas no sólo de riesgo de desastres, sino de poca resiliencia para hacer frente y recuperarse de una eventualidad de esa naturaleza, porque aunada al analfabetismo generalmente se encuentra una condición socioeconómica precaria.

El mejoramiento de los niveles educativos no es suficiente para reducir la vulnerabilidad y el riesgo de desastres, si se toma en cuenta la existencia de empleos productivos mal remunerados, las actividades productivas en deterioro y la división de roles socioculturales. La educación es un elemento fundamental para disminuir la vulnerabilidad de las poblaciones costeras, porque les permite adaptarse a nuevas opciones de empleo y obtención de medios de vida en otros sectores, así como afrontar con más capitales de vida los embates de un impacto meteorológico. La educación debe centrarse en el desarrollo no sólo de conocimientos teóricos e históricos, sino también en el de habilidades y capacidades para enfrentar las condiciones de fragilidad y aumentar el empoderamiento social y de género. Los derechos de igualdad en acceso a los recursos pesqueros respaldados por autocontroles de acceso comunitario pueden crear posibilidades de recuperación de las poblaciones de peces e incrementar las de suministro de pescado para el consumo humano y la mejoría de los ingresos en las pesquerías en pequeña escala.

A pesar de que el municipio de Celestún dispone de una gran riqueza cultural y elevada biodiversidad, sus habitantes padecen una serie de problemas de pobreza y falta de acceso a las oportunidades del desarrollo. Una vez que contamos con este acercamiento preliminar basado en fuentes de información secundaria, se propone profundizar en el conocimiento del municipio desde otra perspectiva, a través de la propia voz y sentir de sus habitantes. En el próximo apartado de este documento se proporcionarán datos empíricos tomados de encuestas y entrevistas de percepción socioambiental sobre vulnerabilidad ante huracanes.

\section{PERCEPCIONES SOCIALES SOBRE VULNERABILIDAD EN CELESTÚN}

El estudio de caso que se presenta a continuación documenta la percepción de pobladores del municipio costero de Celestún respecto de su vulnerabilidad frente a huracanes y la evaluación de algunos funcionarios de la PCE en relación con la capacidad institucional en Celestún para proteger a sus habitantes de los huracanes. Según Vargas (1994) la percepción consiste en el reconocimiento, la interpretación y la significación para la elaboración de juicios en torno a las sensaciones obtenidas del ambiente físico y social, proceso en el cual intervienen el aprendizaje, la memoria y la simbolización. Lazos (1999) abona al concepto que las percepciones atribuyen características cualitativas a los objetos o circunstancias del entorno mediante referentes que se elaboran desde sistemas culturales e ideológicos específicos construidos y reconstruidos por el grupo social, lo cual permite generar evidencias sobre la realidad.

A pesar de que la percepción de los individuos está fuertemente conformada por el patrón cultural de la sociedad, no es meramente un reflejo de dicho patrón, sino que pasa por un proceso de asimilación y resignificación individual. De ahí que hombres y mujeres que viven en el mismo espacio geográfico y pertenecen a la misma cultura puedan percibir mundos diferentes y valorarlos de manera distinta. Intervienen distintos factores en la conformación de la percepción individual, a saber: la edad, el nivel educativo, el sexo, el poder, la experiencia y la etnia, entre otros. También ocurre que encontremos percepciones similares entre culturas distintas, en la medida en que los individuos compartan intenciones y experiencias. Leemos el mundo, le damos significado y lo valoramos según nuestra idea de él.

Destacaremos las posiciones de las personas respecto de su acceso a la capacitación para hacer frente a huracanes, de su percepción sobre la capacidad para proteger a su familia en caso de un evento extremo y de la gestión de las autoridades municipales 
frente a huracanes. Estos tres aspectos están íntimamente relacionados en la conformación de la vulnerabilidad social ante la amenaza que significan los huracanes y reflejan diferentes niveles de vulnerabilidad: individual, familiar y comunitaria, esta última asociada a la capacidad institucional construida para la protección de la población.

La muestra encuestada no es uniforme en cuanto a su acceso a la educación formal, sino que se distribuye en rangos que van desde el analfabetismo - $10 \%-$ hasta quienes cursaron bachillerato y más - 15\%-, transitando por primaria incompleta $-30 \%-$, primaria completa $-20 \%-$ y secundaria $-25 \%-$. El bajo nivel educativo es relevante, dado que $60 \%$ del total de personas encuestadas cuenta sólo con la instrucción primaria o menos. Esto nos permite inferir la poca capacidad de resistencia de estos actores sociales para hacer frente y recuperarse de posibles desastres causados por huracanes, toda vez que existe una relación directa entre analfabetismo, pobreza y desastre, dado que el bajo nivel educativo en general se asocia a una condición socioeconómica precaria, la cual abona a la falta de resiliencia y mayor vulnerabilidad en situaciones de desastres.

Un aspecto elemental para prevenir o remediar los problemas ocasionados por los huracanes y por tanto reducir la vulnerabilidad es la posibilidad de acceder a cursos de capacitación. Éste es un medio eficaz del que dispone la población para recibir información sobre cómo protegerse de los efectos de los eventos meteorológicos extremos. Se les preguntó a las personas si habían recibido alguna vez cursos de capacitación en la materia y se encontró que apenas $25 \%$ de los encuestados ha accedido a ellos. No existe una diferencia significativa entre el acceso a la capacitación por parte de mujeres y hombres y tampoco entre personas de diferentes niveles educativos. La falta de capacitación sobre cómo protegerse frente a un huracán es un serio problema porque los huracanes son frecuentes en la costa yucateca. El cuadro 1 enlista las declaratorias de desastre y emergencia derivadas de la exposición del municipio de Celestún a los riesgos meteorológicos - amenazas- durante el periodo de
2002 a 2012. Si bien de 2007 a la fecha no han ocurrido declaratorias de emergencia o desastre por el impacto de huracanes, la amenaza está latente, dado que en un periodo de sólo cinco años el municipio estuvo sometido a cuatro situaciones graves. Para alcanzar la declaratoria de desastre las alteraciones en personas, bienes, servicios y medio ambiente deben ser tan intensas que sobrepasen la capacidad de respuesta de la comunidad afectada y requieran apoyo externo. La situación de emergencia también implica serias pérdidas al ser el resultado de eventos adversos de características similares a los desastres, con la diferencia de que la comunidad afectada está en la capacidad de controlarla por sus propios medios.

Aunque la zona tiene alta incidencia de huracanes, la capacitación e información que ha recibido la población sobre cómo resolver los problemas asociados con la ocurrencia de fenómenos extremos ha sido escasa. La presencia de organizaciones sociales y de universidades en procesos de capacitación no está registrada en las respuestas de las personas encuestadas. La situación es preocupante porque en la región actúan varias organizaciones sociales de protección de los manglares, entre otras, y en Sisal - localidad muy cercana a Celestún, perteneciente al municipio de Hunucmá- hay un campus de la Universidad Nacional Autónoma de México (UNAM). A pesar de la magnitud de los daños causados por los huracanes a las poblaciones, y de la posibilidad real de que esta tendencia se agudice debido a los impactos negativos del cambio climático, el problema de los desastres no ha sido debidamente incorporado en la agenda educativa y comunicativa de las organizaciones regionales y tampoco en los planes de desarrollo económico y social del municipio.

Se preguntó a las personas encuestadas cuál es su percepción acerca de su capacidad para protegerse y proteger a su familia en caso de una amenaza, como un huracán. Contra lo que se pudiera esperar, dado el limitado acceso a cursos de capacitación, la mayoría - ocho de cada diez- afirmó sentirse capaz de emprender acciones para protegerse a sí mismo y a su familia de una amenaza meteorológica. Se observa 
Cuadro 1. Declaratorias de emergencia o desastre en Celestún debido a huracanes

\begin{tabular}{lcl}
\hline \multicolumn{1}{c}{ Fecha de ocurrencia } & Tipo de declaratoria & Evento \\
\hline 21 de agosto de 2007 & Desastre & Huracán Dean \\
\hline 17 y 18 de julio de 2005 & Desastre & Huracán Emily \\
\hline 16 de julio de 2005 & Emergencia & Huracán Emily \\
\hline 20 y 22 de septiembre de 2002 & Emergencia & Huracán Isidore \\
\hline
\end{tabular}

Fuente: "Declaratorias de emergencia de desastre y contingencia para ciclones tropicales" (Cenapred).

una diferencia cuando se analiza la percepción de los hombres y de las mujeres en relación con su capacidad para salvaguardar a sus familias y a sí mismos de los embates de los huracanes. Las mujeres se sienten menos capaces de brindar protección que los hombres -70\%-, mientras que alrededor de $90 \%$ de éstos piensa que puede dar protección a los suyos. Recordemos que no hay diferencia por sexo en la recepción de cursos de capacitación. La explicación de esta divergencia podría corresponder a los roles de género, los cuales determinan comportamientos y actividades diferenciadas entre hombres y mujeres, con una valoración social jerárquica de las actividades consideradas masculinas en comparación con las femeninas, lo que incide en la reducción de la autoestima de las mujeres y en la negación de sus propias capacidades para hacer frente a las amenazas.

Si sumamos a la baja autoestima de las mujeres el hecho de que constituyen $70 \%$ de la población que vive en extrema pobreza en el mundo (Spiller, 2006), podemos inferir que las mujeres pobres son más vulnerables que los hombres, dado que la vulnerabilidad está íntimamente relacionada con el desarrollo social y económico. A mayor vulnerabilidad, mayor el riesgo de desastres, entonces la población pobre femenina será la más afectada por los huracanes y la que dispondrá de menores recursos en esas situaciones. Al igual que la percepción diferenciada entre hombres y mujeres, detectamos que a menor nivel educativo las personas perciben que tienen menos capacidad para protegerse a sí mismas y a sus familias de los embates de los huracanes: $62 \%$ de los encuestados que no ha tenido acceso a mayores niveles de estudios formales contra 95\% de quienes cursaron hasta el bachillerato o más. Una posible ruta de análisis para una diferencia tan significativa entre las respuestas de acuerdo con el acceso a niveles de educación formal puede ser la seguridad que brinda contar con estudios asociada a la creencia de que los conocimientos adquiridos en la escuela serán útiles frente a la emergencia de un huracán.

Para conocer la fortaleza o debilidad de las autoridades municipales ante este tipo de contingencia se preguntó a la población si considera que las autoridades municipales tienen la capacidad para prevenir y atender esos fenómenos. Al respecto, la población tiene una opinión dividida, dado que más de la mitad de las personas dio una respuesta negativa $-58 \%$ en tanto que $42 \%$ piensa que hay capacidad institucional local construida para prevenir y remediar los efectos de huracanes. Las percepciones son diferenciadas según el grado de escolaridad: mientras más de $60 \%$ de los encuestados con menor acceso a los estudios formales - sin estudios, primaria incompleta, primaria completa y secundaria- no cree en la capacidad institucional local instalada para hacer frente a los huracanes, menos de $40 \%$ de las personas que cursaron el nivel de bachillerato o más comparten la misma percepción. Estas respuestas son contrarias a lo que podría esperarse, en la medida en que un mayor nivel educativo puede llevar a una actitud más crítica respecto de la actuación del municipio, por el nivel de conocimiento 
Figura 1. Sistema Municipal de Protección Civil

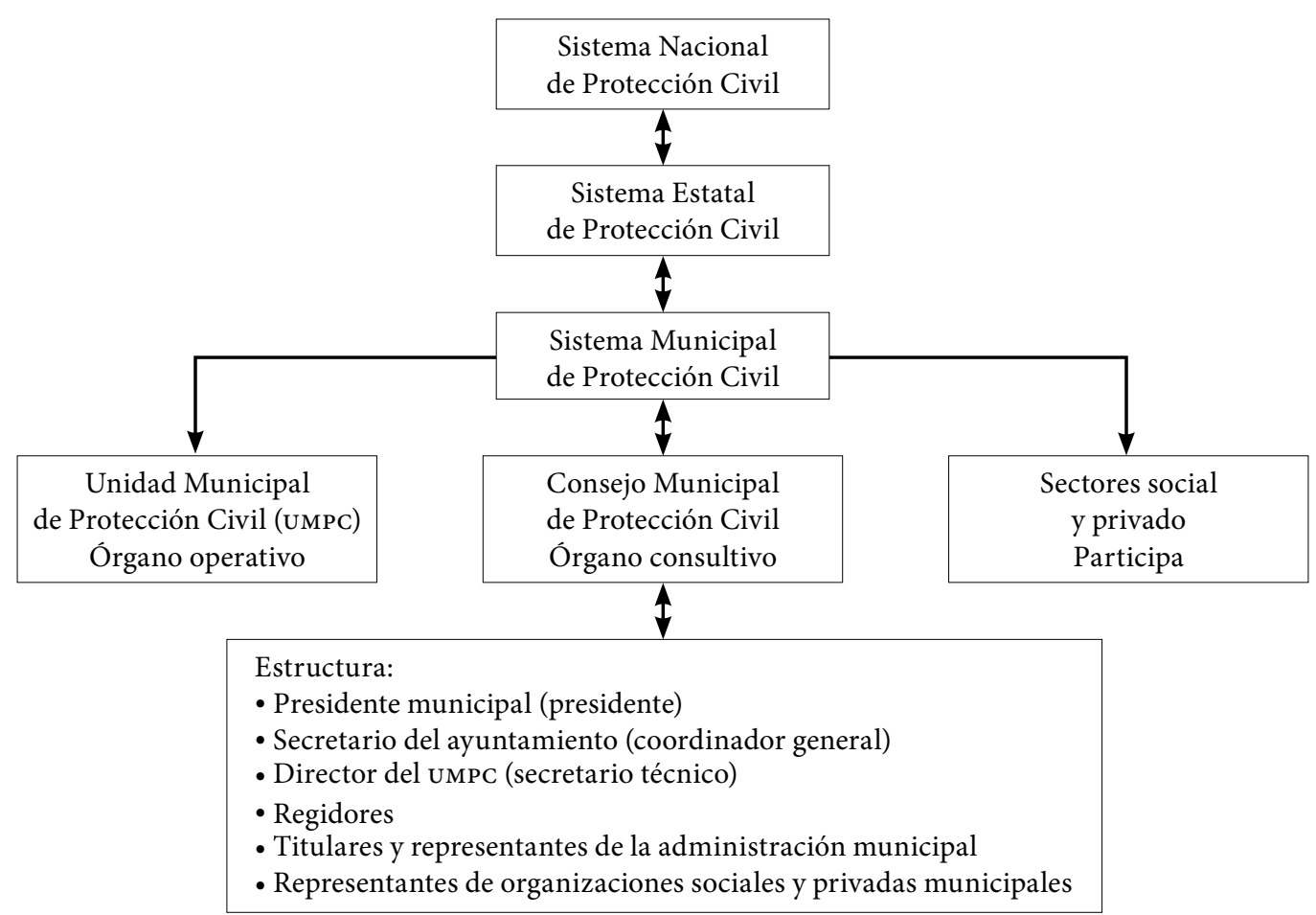

Fuente: Elaboración propia.

adquirido y la percepción de que se hace menos de lo que se debería, pero también puede significar que las personas con mayor nivel educativo tienen mayor acceso a los planes y programas del ayuntamiento y mantienen una comunicación más cercana con éste, lo que afianza la credibilidad en las capacidades institucionales. Según la Ley de Protección Civil del Estado de Yucatán (Gobierno del Estado de Yucatán, 1999) el Sistema de Protección Civil municipal debe estar integrado por el Consejo Municipal, la Unidad Municipal y los representantes de los sectores público, social y privado. Los Consejos de Protección Civil constituyen:

un órgano consultivo de coordinación de acciones y de participación social para la planeación de la protección en el territorio municipal, y será el responsable de propiciar la participación de los sectores de la sociedad a fin de integrar el Sistema Municipal (Gobierno del Estado de Yucatán, 1999: 10).
Con base en el artículo 38 de dicha ley, los Consejos de Protección Civil se integran conforme a la siguiente estructura: presidente del consejo: presidente municipal; coordinador general: secretario del ayuntamiento; secretario técnico: director de la Unidad Municipal de Protección Civil (UMPC); regidores del ayuntamiento; titulares y representantes de las dependencias y entidades de la administración pública municipal cuya área de competencia corresponda a los objetivos del Sistema Municipal de Protección Civil, y representantes de las organizaciones sociales y privadas de carácter municipal.

Por su parte, las UMPC tienen como función: "elaborar, instrumentar, dirigir y operar la ejecución de los programas en la materia, coordinando sus acciones con las dependencias, instituciones y organismos del sector público, social y privado de carácter municipal" (Gobierno del Estado de Yucatán, 1999: 11). El sector social se organiza en grupos de voluntarios 
que participan en las tareas de emergencia y rescate. El sector privado proporciona de manera voluntaria vehículos, instalaciones e insumos o establece convenios. La figura 1 muestra la conformación del Sistema Municipal de Protección Civil. La primera instancia para actuar en caso de emergencia corresponde a la autoridad municipal de Protección Civil. En caso de que la emergencia supere la capacidad de respuesta de esta instancia, se acude a la autoridad estatal. Si ésta resulta insuficiente, se procede a informar a las instancias federales. Si bien está normada y reglamentada la actuación de los municipios ante los eventos meteorológicos extremos, la práctica cotidiana de las administraciones municipales no refleja esta organización en términos de prevención y atención de los riesgos de desastres y ello se traduce en las percepciones sociales sobre la gestión de las autoridades ante crisis de esta naturaleza. Un nudo importante para el desempeño de la UPCM es la rotatividad de su director. En el caso de Celestún, el director de Protección Civil fue nombrado por la administración en curso. Un puesto crucial para la sustentabilidad del municipio se asigna con criterios de afinidades personales y políticas de la autoridad en turno. De acuerdo con el punto de vista de un funcionario de la PCE, la breve permanencia de los directores municipales de Protección Civil es uno de los mayores obstáculos para avanzar de manera sólida en atención de desastres:

Ése es un gran problema: los cambios. El problema más grande que nosotros tenemos con ellos, porque cuando nosotros capacitamos a los directores y ya podemos trabajar a un nivel más alto, los cambian. Inclusive se les trae capacitación de la ciudad de México, de Cenapred, de la Coordinación General, pero cuando ellos ya están capacitados se van (testimonio de funcionario de PCE).

La percepción sobre los problemas que implica un cambio constante del personal de la UPCM es compartida por varias de las personas entrevistadas. Una de ellas manifiesta: "Cada administración cambia. Si hubiera siempre un mismo grupo que llevara una línea, porque me imagino que reciben talleres, preparación". La debilidad institucional de Protección Civil municipal no es exclusiva de Celestún, también se advierte en otros municipios, y no sólo de Yucatán, sino de diversas entidades de la República Mexicana. Al respecto, personal de PCE afirma que hay directores municipales de Protección Civil que no saben leer ni escribir, de edad avanzada, quienes lejos de poder ayudar a la gente tienen que ser atendidos en caso de emergencia o a quienes se les dificulta el uso de herramientas de cómputo, así como la redacción de documentos o el llenado de formatos, porque son personas de campo. De ahí que se haya solicitado al Congreso del estado que la Dirección de Protección Civil a nivel municipal sea un cargo de carrera, iniciativa en la que se está trabajando desde hace dos años.

Es evidente que algunos puestos municipales de extrema relevancia para la sustentabilidad del municipio, como la UMPC, son otorgados como pago a apoyos prestados y no con base en un perfil profesional de habilidades, capacidades o competencias. Ante cada cambio de administración municipal PCE se ve obligada a fortalecer las capacidades de los directores a través de cursos de inducción y de una relación permanente en la que se les asesora, supervisa, capacita y monitorea para el cumplimiento de sus funciones y responsabilidades, entre las que se encuentran: elaborar el plan municipal de contingencias, en el que se establecen las acciones que deben seguirse para cada agente perturbador: incendios, lluvias, huracanes, etc.; la instalación de los Consejos Municipales para las temporadas de incendios y huracanes, y la preparación de atlas de riesgo.

Pese a este esquema, la coordinación y colaboración de los ayuntamientos con PCE no es la deseable. Por ejemplo, personal de PCE menciona que no se reportan los cambios de directores de Protección Civil para actualizar el directorio estatal en caso de emergencia, que no se cumple en tiempo y forma con los procedimientos para la elaboración de los planes de emergencia o la instalación de los Consejos Municipales, o bien que no se autoriza, supervisa ni 
promueve la asistencia de los directores a las reuniones mensuales. Otro problema detectado, relacionado de manera directa con el compadrazgo para la selección de quien ocupará el puesto de director de Protección Civil municipal, es la falta de compromiso y vocación de servicio en algunas de las personas designadas para dicho cargo. Todo esto contribuye a delinear una percepción ciudadana que no aprueba del todo la gestión de las autoridades municipales ante los eventos extremos.

Afortunadamente, no todos los municipios de la costa yucateca emplean estas prácticas clientelares en la definición del personal que ocupa puestos clave en la administración municipal. Un ejemplo de ello es San Felipe, donde la misma persona desempeña el cargo de director de Protección Civil desde 2003 y goza de un amplio reconocimiento en el pueblo como un funcionario capaz y ejemplar, además de que posee un gran poder de convocatoria. De hecho, su liderazgo ha permitido la conformación de una brigada antihuracanes de casi 35 personas, quienes prestan trabajo voluntario. Sin embargo, San Felipe es la excepción a la regla y los municipios tendrían que avanzar hacia allá para construir instituciones sólidas, sin rotación de personal en cada elección de presidencia municipal, con profesionales comprometidos y capacitados en el tema, pues sólo así se logrará reducir la vulnerabilidad institucional para hacer frente a las amenazas.

\section{CONCLUSIONES}

Los aspectos sociales y ambientales de los riesgos, las respuestas individuales e institucionales frente a los desastres, así como la naturaleza de las relaciones entre desastres y desarrollo han sido objeto de una creciente atención por parte de investigadores de las ciencias sociales porque los riesgos de desastres se han convertido en una amenaza tanto para las sociedades menos desarrolladas como para las industrializadas y avanzadas, en virtud del cambio climático. Si bien ya se cuenta con un relevante avance en la comprensión de dichos temas, hay todavía numerosas cuestiones a las que la investigación social debe intentar responder para comprender los procesos de interacción entre las sociedades y su medio ambiente y la relación de ésta con los desastres. El municipio de Celestún posee una serie de características que lo hacen vulnerable a los desastres por el impacto de huracanes, entre ellas su ubicación, las condiciones de las viviendas y el deterioro de los ecosistemas costeros que sirven de barrera natural contra los huracanes y tormentas tropicales. Además de estas circunstancias de fragilidad física ante los fenómenos meteorológicos, la situación social, económica e institucional del municipio agrega elementos de vulnerabilidad a los riesgos, con la continua amenaza de desastre. El fuerte crecimiento poblacional de los últimos años, carente de un proceso de planeación urbana y de los servicios públicos, es otra faceta de su vulnerabilidad.

Si bien es evidente la vulnerabilidad del municipio frente a fenómenos hidrometeorológicos extremos, manifiesta no sólo en la dimensión física sino en la socioambiental, también es indiscutible la poca importancia que el municipio brinda al tema, es decir, su vulnerabilidad institucional. Una conclusión fundamental a la cual se ha llegado con el desarrollo de este capítulo es que la falta de capacitación de la población en el manejo de riesgos, aunada al muy bajo perfil de Protección Civil municipal, contribuye a que, por una parte, la sociedad no perciba el peligro como tal y, por otra, a que las normas y programas de prevención, atención y recuperación de desastres sean escasas y no se les otorgue la relevancia debida, lo que genera vulnerabilidad en una espiral de riesgo para la sociedad. Es primordial que se diseñen políticas públicas y programas de prevención social de riesgos en escuelas, comercios y empresas en Celestún. Que la UMPC se fortalezca y sea una instancia independiente del municipio a fin de que no cambie sistemáticamente su director, que de manera participativa se elaboren mapas de riesgo en sus tres etapas - prevención, atención y recuperación- y que estas iniciativas sean consideradas en los planes 


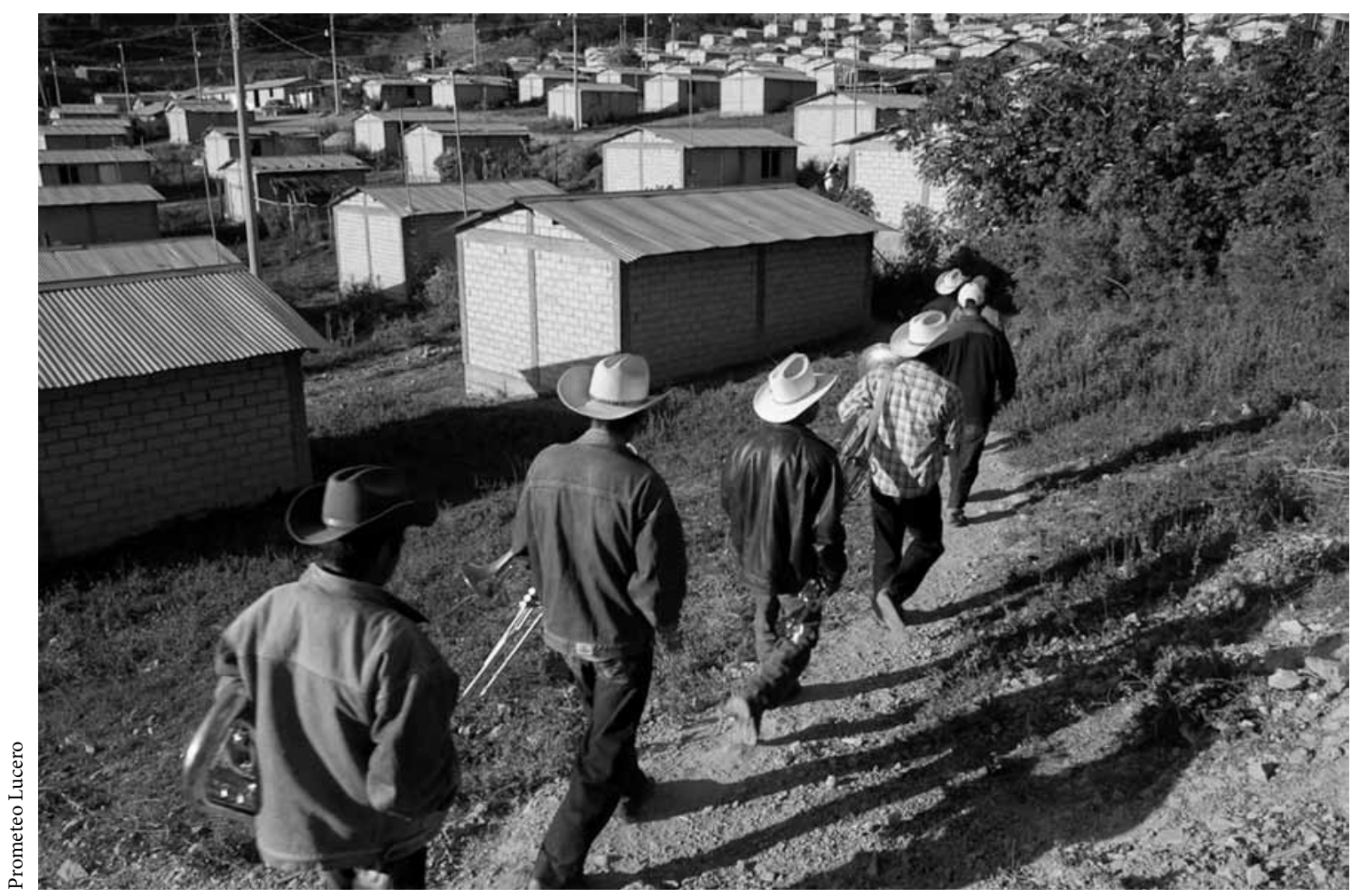

La Barca, pueblo de La Montaña de Guerrero construido después de que un sismo acabara con las viviendas.

municipales de desarrollo. Es decir, el municipio debería plantear una agenda de temas estratégicos relacionados con el manejo de riesgos de desastres, que defina áreas de intervención articuladas entre las políticas sociales y la gestión ambiental.

Es imperativo fortalecer los procesos de gestión de riesgos no sólo en el municipio de Celestún, sino en el estado de Yucatán y en todo el país, con la finalidad de generar condiciones para disminuir la vulnerabilidad. Cabe subrayar en este aspecto que la inversión en la reducción de la vulnerabilidad repercute de manera directa en los montos de las inversiones posteriores a un desastre frente a eventos extremos, como los huracanes. Los huracanes amenazan el desarrollo del municipio: destruyen años de esfuerzo e inversiones, crean nuevas cargas a la sociedad para la reconstrucción y desvían las prioridades de desarrollo de los objetivos a largo plazo hacia la satisfacción de necesidades más inmediatas.

Como gobierno y como sociedad deben analizarse los impactos causados por los huracanes en el municipio y aprovechar esta información con el fin de hacer acopio de las lecciones aprendidas. A partir de esto pueden definirse lineamientos de políticas estratégicas e instrumentos de gestión ambiental y social para reducir en el futuro la vulnerabilidad a fenómenos similares, al incorporar las medidas preventivas más adecuadas al actual proceso de reconstrucción. Es de vital importancia la creación de un marco institucional adecuado, que disponga de mecanismos mediante los cuales puedan ponerse en práctica medidas de reducción de la vulnerabilidad. La piedra angular de este marco es un sistema participativo en el que todos los sectores - gubernamental, privado, sociedad civil- adopten medidas para prevenir y reducir la vulnerabilidad ante 
amenazas naturales. La educación ambiental brinda a las comunidades no sólo la destreza necesaria para tomar decisiones con base en información suficiente, sino también la motivación para participar y asumir la responsabilidad de la gestión ambiental.

\section{BIBLIOGRAFÍA}

Adger, W. Neil, 2006, "Vulnerability", en Global Environmental Change, vol. 16, núm. 3, University of London, pp. 268-281.

Anderson, Mary y Peter Woodrow, 1989, Rising from the Ashes, Development Strategies in Time of Disaster, Westwiew Press, Boulder.

Barrachena, Julieta et al., 2000, "Una propuesta metodológica para el estudio de la vulnerabilidad social en el marco de la teoría social del riesgo", ponencia, IV Jornadas de Sociología, Facultad de Ciencias Sociales, Universidad de Buenos Aires, Buenos Aires.

Barriga Machicao, Milka Mabel, 2004, "El rol del capital social en la reducción de vulnerabilidad y prevención tesis de magíster en socioeconomía ambiental, Centro Agronómico Tropical de Investigación y Enseñanza, Programa de Enseñanza para el Desarrollo y la Conservación, Turrialba.

Batllori, Enrique, 2003, Estudio técnico para el desarrollo del ordenamiento ecológico del territorio para la Reserva de la Biosfera Ría Celestún, Centro de Investigación y Estudios Avanzados, Mérida.

Blaikie, Piers et al., 1998, Vulnerabilidad: el entorno social, político y económico de los desastres, Red de Estudios Sociales en Prevención de Desastres en América Latina, Intermediate Technology Development Group, Bogotá.

Camarasa Belmente, Ana María y Fernando Moreno Sanz, 1994, Algunas reflexiones sobre la percepción del cambio climático en una muestra de población adulta de nivel cultural medio, Universidad de Alcalá (Serie Geográfica, vol. 4), Madrid.

Canto Sáenz, Rodolfo, 2001, Del henequén a las maquiladoras, la política industrial en Yucatán 1984-2001, Instituto Nacional de Administración Pública, Universidad Autónoma de Yucatán, Mérida.

Centro Nacional de Prevención de Desastres (Cenapred), 2012, "Declaratorias de emergencia de desastre y contingencia para ciclones tropicales", Centro Nacional de Prevención de Desastres, en línea: <http://atl.cenapred.

unam.mx/index.php?option=com_docman\&task= cat_view\&gid=51\&Itemid $=215>$, consultado el 15 de junio de 2012.

Comisión Nacional de Áreas Naturales Protegidas (Conanp), 2002, Programa de Manejo Reserva de la Biosfera Ría Celestún, Comisión Nacional de Áreas Naturales Protegidas, en línea: <http://www.conanp.gob.mx/que hacemos/pdf/programas_manejo/celestun.pdf>, consultado el 28 de mayo de 2012.

Consejo Nacional de Población (Conapo), 2011, Índice de marginación por entidad federativa y municipio 2010, Consejo Nacional de Población, México.

Fraga, Julia, 1993, "La inmigración y sus principales efectos en la costa yucateca: estudio de caso en Celestún y Sisal", tesis de maestría, Universidad Autónoma de Yucatán, Mérida.

García Acosta, Virginia, 2005, "El riesgo como construcción social y la construcción social de riesgo", en Desacatos. Revista de Antropología Social, núm. 19, pp. 11-24.

Gobierno del Estado de Yucatán, 1999, Ley de Protección Civil del Estado de Yucatán, en línea: <http://www.yucatan.gob.mx/gobierno/orden_juridico/Yucatan/Leyes/ nr232rf1.pdf>, consultado el 1 de noviembre de 2012.

Instituto Nacional de Estadística y Geografía (INEGI), 2011, Censo de Población y Vivienda 2010, Instituto Nacional de Estadística y Geografía, México.

Lavell, Allan, 1993, "Ciencias sociales y desastres en América Latina: estrategias de intervención”, en Andrew Maskrey (comp.), Los desastres no son naturales, Red de Estudios Sociales en Prevención de Desastres en América Latina, Tercer Mundo Editores, Bogotá, pp. 31-58.

, 1996, "Degradación ambiental, riesgo y desastre urbano: problemas y conceptos", en María Augusta Fernández (comp.), Ciudades en riesgo: degradación ambiental, riesgos urbanos y desastres, Red de Estudios Sociales en Prevención de Desastres en América Latina, United States Agency for International Development, Lima, pp. 21-60.

_, 2000 , "Desastres y desarrollo: hacia un entendimiento de las formas de construcción social de un desastre: el caso del huracán Mitch en Centroamérica”, en Nora Garita y Jorge Nowalski (comps.), Del desastre al desarrollo humano sostenible en Centroamérica, Centro Internacional para el Desarrollo Humano Sostenible, Banco Interamericano de Desarrollo, Costa Rica, pp. 7-45.

, 2005, "Desastres y desarrollo: hacia un entendimiento de las formas de construcción social de un desastre: el caso del huracán Mitch en Centroamérica”, en Armando Fernández (comp.), Comarcas vulnerables: riesgos y desastres en Centroamérica y el Caribe, Coor- 
dinadora Regional de Investigaciones Económicas y Sociales, Buenos Aires, pp. 11-44.

Lazos Chavero, Ma. Elena, 1999, "Utopía y realidad: perspectivas de los acervos de conocimiento agrícolas y ecológicos para el desarrollo rural", en Haydea Izazola (coord.), Desarrollo sustentable, medio ambiente y población, a cinco años de Río, Consejo Estatal de Población, El Colegio Mexiquense, Toluca, Zinacantepec, pp. 101-136.

Leff, Enrique, 1993, "La dimensión cultural del manejo integrado, sustentable y sostenido de los recursos naturales", en Enrique Leff y Julia Carabias (coords.), Cultura y manejo sustentable de los recursos naturales, vol. 1, Miguel Ángel Porrúa, Centro de Investigaciones Interdisciplinarias en Humanidades, México, pp. 55-88.

Luhmann, Niklas, 1992, Sociología del riesgo, Universidad Iberoamericana, Universidad de Guadalajara, Guadalajara.

Macías, Jesús Manuel, 1992, "El significado de la vulnerabilidad social frente a los desastres", en Revista Mexicana de Sociología, núm. 4, Instituto de Investigaciones SocialesUniversidad Nacional Autónoma de México, México.

Oltra, Christian et al., 2009, "Cambio climático: percepciones y discursos públicos”, en Prisma Social. Revista de Ciencias Sociales, núm. 2, Centro de Investigación Sociotécnica, Centro de Investigaciones Energéticas, Medioambientales y Tecnológicas, Barcelona.

Organización de las Naciones Unidas para la Educación, la Ciencia y la Cultura (UNEsco), 2006, Educación para todos. La alfabetización, un factor vital, Organización de las Naciones Unidas para la Educación, la Ciencia y la Cultura, París.

Organización de los Estados Americanos (oEA), 1991, Desastres, planificación y desarrollo: manejo de amenazas naturales para reducir los daños, Organización de los Estados Americanos, United States Agency for International Development, Washington.
Quinceno Montoya, Claudia Nancy, 2005, “Escenarios de una catástrofe”, en Revista de Antropología Iberoamericana, núm. 39, enero-febrero, pp. 1-6.

Saavedra, María del Rosario, 1996, Desastre y riesgo. Actores sociales en la reconstrucción de Armero y Chichina, Centro de Investigación y Educación Popular, Bogotá.

Salles, Vania y Paz López, 2004, Viviendas pobres en México: un estudio desde la óptica de género, Consejo Latinoamericano de Ciencias Sociales, Buenos Aires.

Spiller, Ingrid, 2006, "Por el derecho al agua: la sociedad civil internacional, sus temas y estrategias", en Sophie Esch et al., La gota de la vida: hacia una gestión sustentable y democrática del agua, Fundación Heinrich Boell, México, pp. 142-149.

Uc Espadas, Martha Concepción, 2007, "Estrategias de vida en hogares costeros, estudio de caso en Celestún, Yucatán”, tesis de maestría en ciencias, Centro de Investigaciones y Estudios Avanzados, Mérida.

Uribe, Alberto et al., 1999, "Reducción de la vulnerabilidad ante amenazas naturales: lecciones aprendidas del huracán Mitch, documento estratégico sobre gestión ambiental", Banco Interamericano de Desarrollo, Estocolmo, en línea: <http://www.iadb.org/regions/re2/ consultative_group/groups/ecology_workshop_1esp. htm>, consultado el 11 de octubre de 2011.

Vargas Melgarejo, Luz María, 1994, "Sobre el concepto de percepción”, en Alteridades, vol. 4, núm. 8, pp. 47-53.

Wilches-Chaux, Gustavo, 1989, Desastres, ecologismo y formación profesional: herramientas para la crisis, Servicio Nacional de Aprendizaje, Popayán.

, 1993, "La vulnerabilidad global", en Andrew Maskrey (comp.), Los desastres no son naturales, Red de Estudios Sociales en Prevención de Desastres en América Latina, Tercer Mundo Editores, Bogotá. , 2000, En el borde del caos, Centro Editorial Javieriano, Bogotá. 